\title{
An Asynchronous Data Transmission Method in Heterogeneous Wireless Networks
}

\author{
Changhao Wang $\mathbb{C}^{1,2}$ Shining Li, ${ }^{1}$ Bingqi Li, ${ }^{1}$ and Qianwu Chen ${ }^{1}$ \\ ${ }^{1}$ School of Computer Science and Engineering, Northwestern Polytechnical University, Xi'an 710072, China \\ ${ }^{2}$ Shaanxi University of Science \& Technology, Xi'an 710021, China \\ Correspondence should be addressed to Changhao Wang; wchanghao@mail.nwpu.edu.cn
}

Received 20 August 2020; Revised 8 September 2020; Accepted 15 September 2020; Published 14 October 2020

Academic Editor: Shubo Wang

Copyright (c) 2020 Changhao Wang et al. This is an open access article distributed under the Creative Commons Attribution License, which permits unrestricted use, distribution, and reproduction in any medium, provided the original work is properly cited.

\begin{abstract}
With the massive deployment and application of intelligent systems such as smart life, smart medical care, and smart transportation, it is becoming more common for heterogeneous wireless networks such as WiFi, Zigbee, and Bluetooth to coexist in the same physical space. To improve the communication efficiency under the coexistence of heterogeneous wireless networks, this paper proposes an asynchronous data transmission method based on CTC technology, which constantly monitors the sleep and wake-up cycles of heterogeneous devices, makes full use of the wake-up cycle of the receiving terminal, and uses asynchronous methods to achieve cross-technology transmission of data packets. We also give a specific implementation scheme and use the program to simulate the transmission effect. The results show that our proposed method effectively reduces data transmission time and balances channel transmission throughput.
\end{abstract}

\section{Introduction}

In recent years, smartphones, smart bracelets/watches, wireless sensor network devices, etc. have shown explosive growth, with the increasing popularity of smart devices using various wireless communication technologies in daily life (homes, offices, shopping malls, etc.) and in specific occasions (hospitals, factories, etc.) [1]. It is becoming more common for heterogeneous wireless networks (such as WiFi, Zigbee, and Bluetooth) to coexist in the same physical space and industry control $[2,3]$. People use WiFi networks to access the Internet in their homes or office buildings; Bluetooth devices such as smart speakers and wireless headphones also transmit information through Bluetooth. At the same time, Zigbee protocol-based intrusion prevention systems and building management systems are also deployed in these areas, and different types of wireless networks are rapidly expanding their application space [4-6]. Because the physical layers of these heterogeneous devices are completely different [7] and the modulation and demodulation methods are too different, the wireless communication efficiency of the coexistence environment is low, and the communication performance of the wireless channel is reduced.

The key to solve the coexistence problem lies in the cooperative communication between the coexistence technologies. The appearance of cross-technology communication makes the establishment of good communication links between heterogeneous wireless network devices and exchanges control information across heterogeneous technologies. However, different wireless technologies such as WiFi, Bluetooth, and Zigbee have different communication power. In the coexistence environment of wireless networks, low-power Zigbee, Bluetooth, and other communication technologies will obviously receive interference from highpower WiFi signals. These differences result in the inability to fairly compete and use channels between different wireless communication technologies, resulting in reduced network throughput and increased communication delay.

Under the premise of in-depth research and consideration of existing communication standards, to improve the communication efficiency under the coexistence of 
heterogeneous wireless networks, this paper presents an asynchronous data transfer method named ADT. It makes full use of the wake-up period of different wireless technologies to transmit data asynchronously, thereby improving channel utilization and throughput under the coexistence environment of wireless networks and improving the fair occupation of different channels.

The main contributions of this paper are as follows:

(i) ADT, a CTC-based asynchronous wireless network data transmission method, can effectively improve the system throughput and fairness

(ii) We present an algorithm to dynamically detect the wakefulness and dormancy periods of wireless networks and asynchronously transmit data

(iii) We simulate this transmission method through a computer program and estimate its effect

In the following text, Section 2 discusses the related works. Section 3 describes the design of ADT in detail. Section 4 describes the implementation and the evaluation result. Finally, conclusions are given in Section 5.

\section{Related Works}

Research on coexistence communication of heterogeneous wireless networks involves two aspects: cross-technology communication and data transmission mechanism of wireless networks.

2.1. Wireless Network Data Transmission Mechanism. Wireless devices need to adopt the cotransmission strategy to ensure the communication efficiency under the coexistence of heterogeneous networks. In general, the reliability of data communication is guaranteed at the MAC level, and researchers mainly achieve coexistence transmission by improving the design of the MAC layer. MAC protocol determines the use of wireless channels in heterogeneous wireless networks and is responsible for the allocation and coordination of wireless communication resources for nodes [8]. The MAC protocol can wake up and transmit data only when there is data to be transmitted according to the working status of each node. S-MAC (Sensor-MAC) is a MAC protocol proposed by Wei Ye et al. in the early sensor network research; it uses a synchronization strategy that allows each node to periodically listen and sleep simultaneously to save energy. When less data needs to be sent in each cycle, the S-MAC synchronization strategy will cause a lot of idle monitoring. Later, Joseph et al. proposed b-MAC [9], which adopted the asynchronous leading code strategy to wake up the receiver. It only needs to be slightly longer than a period, and it is guaranteed that the receiver will wake up once during this period; its sleep and wake-up can be performed asynchronously, which significantly reduces the duty-cycle of the MAC protocol (duty-cycle). Buettner et al. proposed the X-MAC protocol [10] that continuously transmits multiple small data packets containing the receiver's link-layer address. Sun et al. proposed the RI-MAC protocol [11], which adopted a receiver-initiated strategy, which effectively solved the phenomenon of conflicts caused by multiple nodes sending data simultaneously in the same wireless domain. These proposed methods and technologies either focus on communication problems of different wireless technologies or focus on system power consumption, without considering the issues of channel occupancy and fairness of transmission under wireless coexistence.

2.2. CTC Techniques. The key breakthrough to solve the coexistence problem lies in the communication and cooperation between coexistence technologies, which requires the exchange of control information across heterogeneous technologies. Chebrolu and Dhekne [12] were the first to study cross-technical communication; they pointed out that heterogeneous technologies with overlap-ping working frequency bands could transmit information through the energy of wireless signals and proposed the first crosstechnology communication scheme Esense. Esense encodes information by the duration of signal energy. Based on Esense, HoWiES [13] introduced a more complete information coding scheme that realizes direct messaging between WiFi and Zigbee. Different from Esense and HoWiES, Zhang and Shin [14] proposed the occurrence mode of GSense modulated wireless signal energy pulse, encoding information through the time interval between adjacent energy pulses. Similar to GSense, FreeBee [15] uses Beacon messages periodically in wireless communication protocols to build cross-technical communication channels and encodes information through the time interval of adjacent beacons. Since Beacon messages account for only a very small part of all messages, the throughput of Beacon-based cross-technology communication is limited, and the performance of cross-technology communication is improved by adjusting the transmission time interval of all messages. In addition, based on the idea of "Morse code," C-Morse [16] conducts cross-technical communication by controlling the length of adjacent data messages. EMF [17] realized one-tomany cross-technology communication by dividing the data message of the sender into multiple layers in time and adjusting the message sending time of each layer. Literature [18] found through experiments that when WiFi sends UDP data packets at a higher rate, the signal energy detected by Zigbee nodes is relatively strong, while when UDP data packets are sent at a lower rate, the signal energy is relatively weak. By adjusting the UDP message transmission rate, the authors use the strength of signal energy to transfer information, to realize direct communication between $\mathrm{WiFi}$ and Zigbee. Wizig [19] realized direct cross-technical communication between $\mathrm{WiFi}$ and Zigbee at a high rate by combining the sending time and sending power of the disturbed packet. Different from the modulation of cross-technical communication using existing packets, B2W2 [20] achieves cross-technical communication from Bluetooth to WiFi by interfering with CSI information at the WiFi receiver terminal. Webee [21] and PMC [22] emulated the Zigbee signal by using $\mathrm{WiFi}$ at the physical layer, improving the throughput of direct communication between $\mathrm{WiFi}$ and Zigbee by at least 3 orders of magnitude, realizing the 
simultaneous communication between the sender end of $\mathrm{WiFi}$ and Zigbee and improving the spectrum utilization ratio significantly.

\section{ADT Design}

In an environment where heterogeneous wireless networks coexist, such as the smart home scene, WiFi and Zigbee are deployed in the same space $[23,24]$, and WiFi communication power is high, which causes communication interference and suppression to Zigbee. However, Zigbee has many nodes, so nodes closer to the coexistence technology can be used as relays to realize data forwarding or communication. As shown in Figure 1, in such a network coexistence scenario, $1 \mathrm{TX}, 1 \mathrm{WiFi} \mathrm{AP}$, and 2 Zigbee nodes together form a data exchange network.

Due to the use of different network protocols and encoding technologies, communication messages of one technology are difficult to decode by communication equipment of another technology. Secondly, some technologies use lower communication power for low power consumption, and the transmission range of communication signals is limited, which is difficult to be detected by the coexistence technology, which further increases the complexity of the problem. In wireless communications, RF chips modulate electromagnetic signals based on data to be sent. For data information $x$, the modulated wireless signal can be expressed as

$$
s[x]=A_{s} e^{i \theta_{s}[x]} .
$$

Among them, $A_{s}$ represents the amplitude (signal energy) of the modulation signal (electromagnetic wave), and $\theta_{s}[x]$ represents the phase of the modulation signal. Propagating through the wireless channel, the signal received by the receiving end can be expressed as

$$
r[x]=H \cdot s[x] .
$$

$H$ is the influence coefficient of wireless channel on signal. Consider the noise and cross-technology interference in the coexistence environment; the signal received can be expressed as (among them, $i[x]$ represents the interference signal of $x, H$ is the channel coefficient where the interference signal is located, and $n[x]$ is the environmental noise superimposed on $x$ ):

$$
r[x]=H \cdot s[x]+H^{\prime} \cdot i[x]+n[x] .
$$

When the wireless communication is interfered, the energy of the interfered signal and the normal communication signal is superimposed with each other as follows:

$$
E\left[|r[x]|^{2}\right]=E\left[\left|H s[x]+H^{\prime} i[x]\right|^{2}\right] .
$$

Cross-technical interference can be determined by detecting the Received Signal Strength (RSS) of the wireless communication. Using this phenomenon, the coexistence technology realizes heterogeneous network perception and transmits communication requirements by detecting communication features of cross-technology interference.

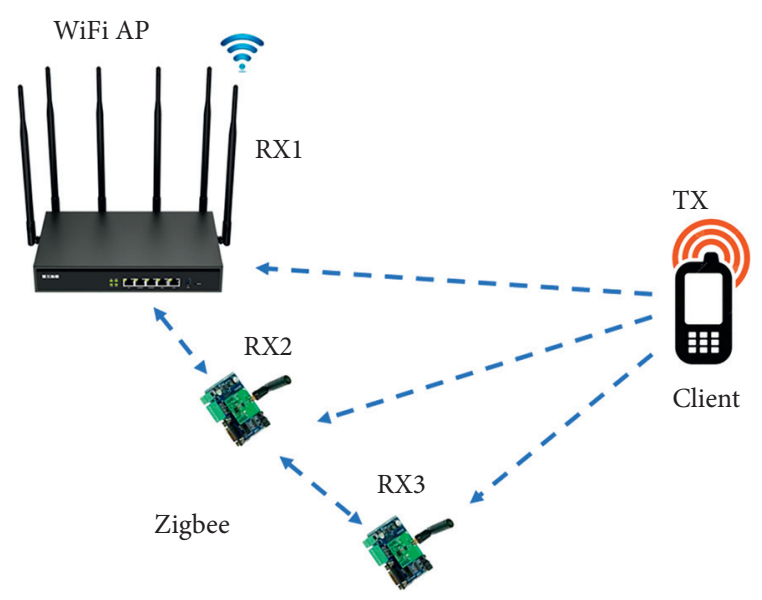

FIgURE 1: Heterogeneous wireless networks coexisting and communication.

3.1. Coexisting and Communication. With the support of CTC technology, TX clients can exchange data with WiFi AP, or with Zigbee nodes RX2 and RX3; when WiFi causes interference to node RX2, RX3 can be used as a relay to exchange data with RX2. If the TX side has data transmission requirements, it can send data packets to RX1, RX2, and RX3. As we know, in order to reduce power consumption, wireless devices will sleep and wake up periodically, and only during the wake-up period can data transmission be completed. When the TX end has a data transmission request to $\mathrm{RX} 1, \mathrm{RX} 2$, and $\mathrm{RX} 3$, it will detect whether the receiving end is in the wake-up period. If it is in the wake-up period, it will transmit data. If it is in the sleep period, it will wait until it wakes up and sends data. If the TX end sends a data packet to the WiFi AP which is in the dormant period-but at the same time, there are other receiving nodes in the wake-up period but cannot transmit data-the data packet will still be in a waiting state until the corresponding device wakes up to complete the data packet transmission. This will cause data transmission delays, channel congestion in local equipment, and imbalance channel utilization [25-27].

3.2. Packet Definition. To implement the method we designed, we define some of this information in the packet we want to send [28-30], As shown in Figure 2, there are four pieces of information in a packet, including beacon, number, data, and flag. Beacon represents whether the current device is in the wake period; before transmitting data, it detects the working state of the receiving end. When the receiving end is in the wake period, it sets the beacon as ' $Y$ '. Otherwise, the receiving end is in sleep period, while beacon is the ' $N$ '. Num represents the current destination receiver number. Data represents the data to be transferred. We set the flag indicating data transmission status; when this data is successfully sent, the flag is set to ' 1 '. If the receiving end of the packet is still in sleeping period, the flag is set to 0 , which indicates we need to wait.

3.3. ADT Method. Based on the above problems, we proposed an asynchronous wireless network data transmission method. In the coexistence scenario shown in Figure 1, TX 


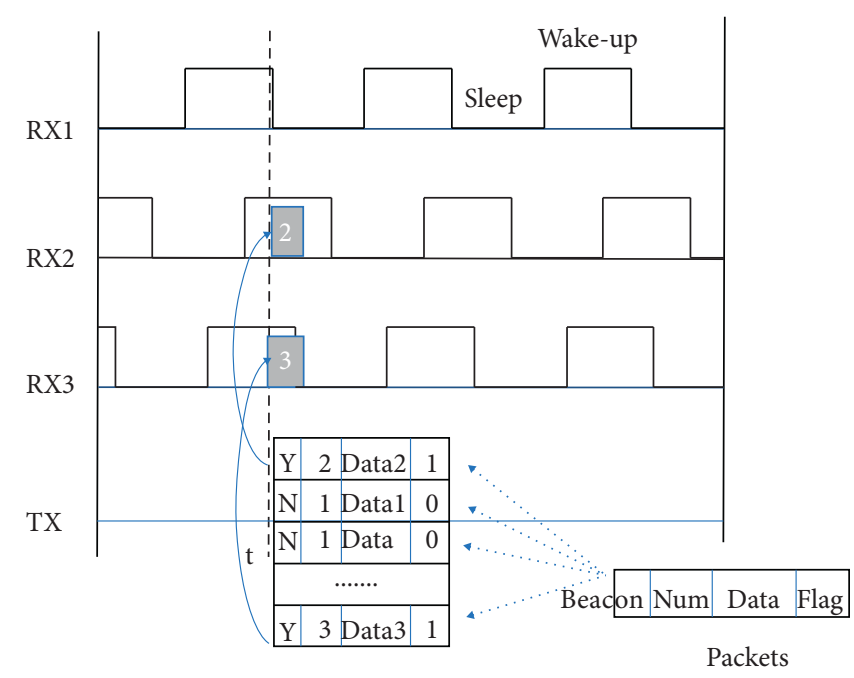

Figure 2: ADT method.

node serves as the data sending terminal, and RX1, RX2, and RX3 serve as the data receiving terminal. Different from the above method, there are $n$ packets, and the current order of the packets is $n_{1}, n_{2}, \ldots, n$. When the TX node sends these data packets, it constantly detects the working status of the receiving end. According to the order of the data packets, if the destination state of the $\mathrm{n} 1$ data packet is the awake state, then send it; if it is the sleep state, it will be placed in the monitored state. At the same time, continue to detect the destination receiver of $n 2$ packet; if awakened, send it, and so on. When the destination receiver of the packet that has been shelved before is in the wake period, the packet shall be sent in time, or the destination receiver is still in the sleep state. But other receivers are in the wake period and have no receiving task. The CTC technology can be used to carry out cross-technology forwarding until all packets are sent. By adopting this method, the receiving end in the wake period can receive or forward the packet in time, while the receiving end in the dormant period can send and receive the packet in time after the waking up, or forward the packet through CTC technology, which can not only solve the packet delay, but also balance the channel and make fair use of it, as shown in Figure 2.

\section{Implementation and Evaluation}

It can be seen from the ADT method that there are two aspects to consider when we want to implement asynchronous data transmission. On the one hand, we need to get the status information of the receiving side to confirm whether it is in the wake-up period. On the other hand, we need to adjust the transmission order of packets according to the state of the receiving end to ensure that each packet can be sent in time. The algorithm is as follows.

4.1. Algorithm. To assess the effectiveness of our approach, we compared it with the traditional method (TRA) and estimated their respective elapsed time using a computer simulation (Algorithm 1).
4.2. Simulation. It is assumed that the receiving terminal periodically sleeps and wakes at intervals of $100 \mathrm{~ms}$ and receives data only in the wake-up state. The receiving terminal RX1, RX2, and RX3 all start to periodically sleep and wake up from a random moment. The sending end is ready to send data packets at a random time $t$. The longest sending time for each data packet is $4 \mathrm{~ms}$. The data packet sequence is randomly generated and corresponds to the receiving terminal.

In the TRA method, at the current sending time of the sender end while the receiver end is in the wake-up period (working interval), the reception can be successful; otherwise, wait until the wake period to receive. Only after the previous packet has been sent can the next packet be sent, and so on, until the end of sending.

In ADT method, the sending terminal sends at time $t$, monitoring the receiver's working status beacon is " $Y$ ", and the receiving terminal is in the wake period (working interval); then it can receive. If the receiving terminal receives successfully, there will be feedback, and the flag is the number one, indicating that the packet is sent successfully. The data packet transmission is carried out in order. First, data packet 1 is sent. If the corresponding receiving terminal is in the working area, the transmission is successful. Otherwise, to detect that other receivers are at working interval and idle, it may transmit data through other receivers under the support of CTC technology. If not at working interval or not available, it is placed in the waiting sequence and requeued; at the same time, data packet 2 is sent instead. If data packet 2 is at its corresponding receiving terminal in the work area, the transmission is successful; otherwise, it will still reenter the end of the queue and proceed in sequence until all data packets are sent.

4.3. Evaluation. We have counted the total elapsed time of sending data packets each time when the number of data packets gradually increased from $0,50,100$, etc, to 1000 in two cases; the results are shown in Figures 3 and 4. 


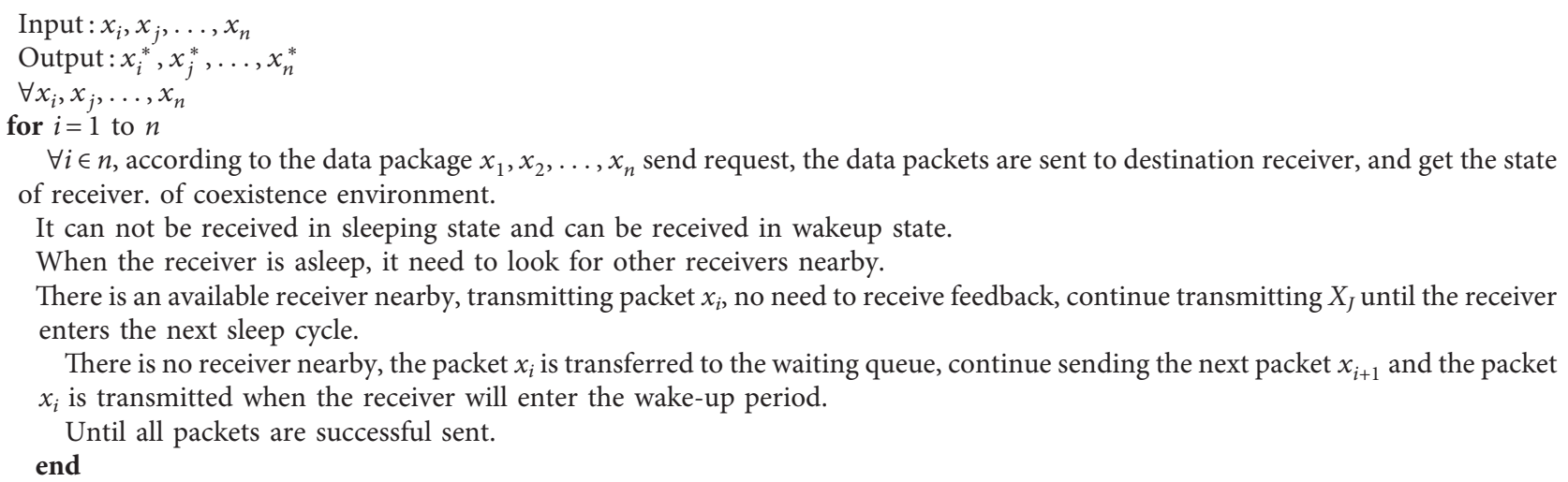

Algorithm 1: Asynchronous transmission algorithm.

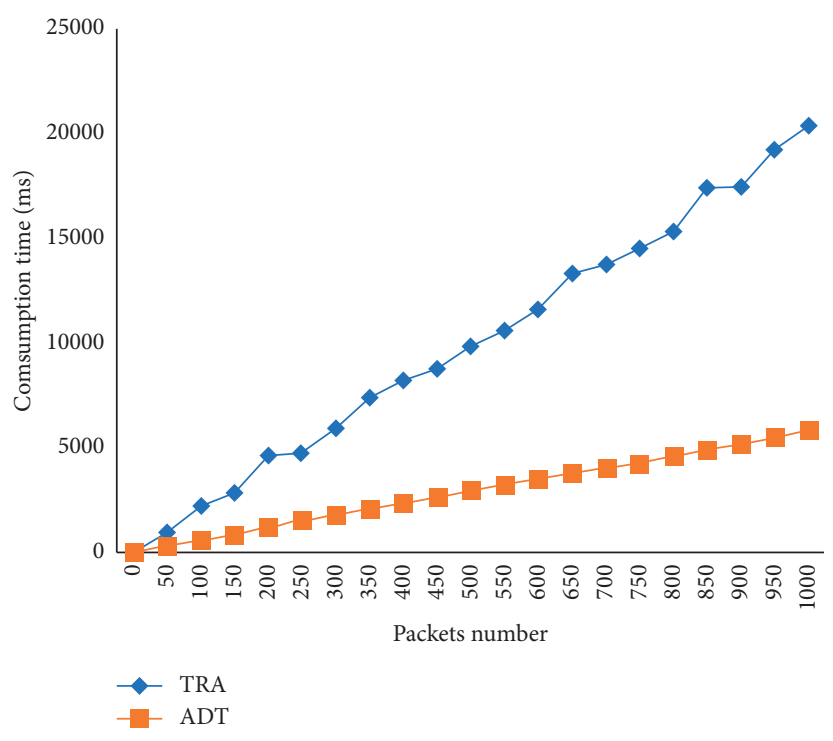

FIGURE 3: Data transferring consumption time.

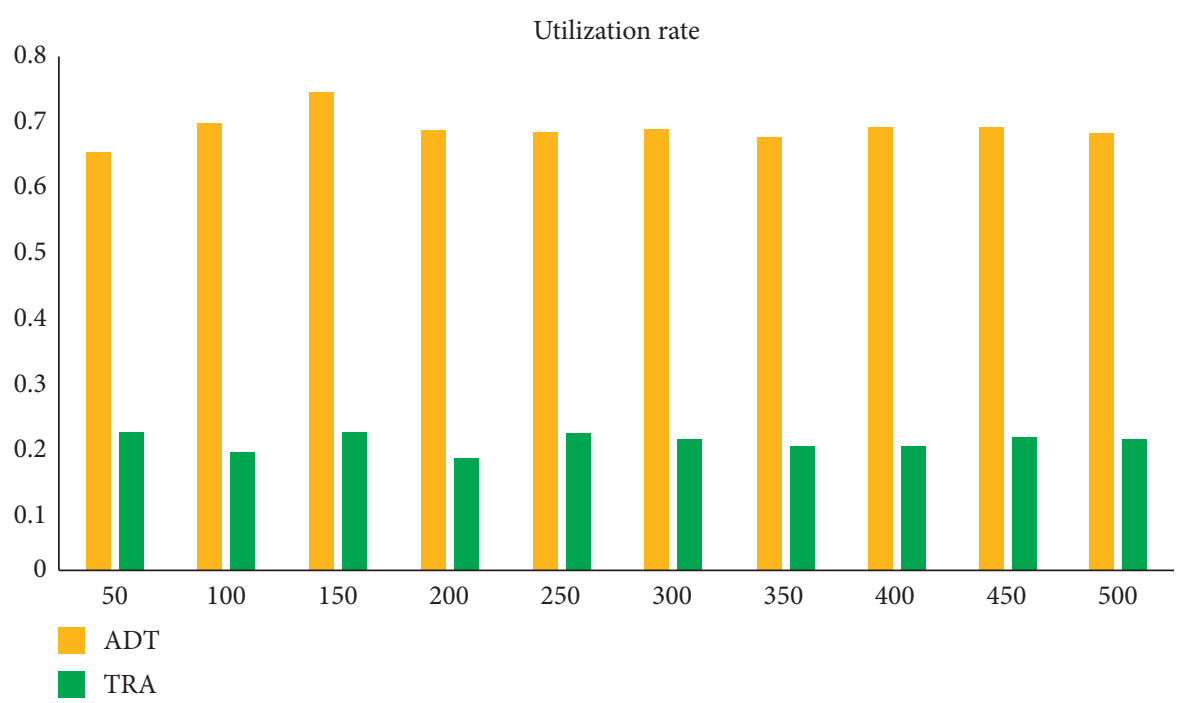

FIgURE 4: Data transferring channel utilization. 
As can be seen from the figures, when the number of packets is small, the time spent on data transmission is not obvious. When the number of data packets increases, the time consumed by TRA method is more than 3 times higher than that consumed by ADT. This result also shows that ADT method can effectively improve the system throughput and reduce the consumption time, thus achieving the goal of balancing channel and saving energy consumption.

\section{Conclusions}

In this paper, we review the methods of data communication and transmission under the coexistence of heterogeneous wireless networks and propose an asynchronous data transmission method based on CTC technology. We describe the design ideas and application scenarios of this method in detail; the data transmission time is estimated by a computer program and compared with the traditional method. The results show that the ADT method can effectively balance the channel and reduce the transmission time. However, this is just some of the work we are currently doing. In the future, how to reduce energy consumption while reducing time consumption is the challenge we face.

\section{Data Availability}

The data used to support the finding of this study are available from the corresponding author upon request.

\section{Conflicts of Interest}

The authors declare that they have no conflicts of interest.

\section{Acknowledgments}

This work was supported in part by the National Natural Science Foundation of China (NSFC) under Grant no.61872434 and the National Key Scientific Research Project of China under Grant no. MJ-2018-S-33.

\section{References}

[1] B. Cheng, M. Wang, S. Zhao, Z. Zhai, D. Zhu, and J. Chen, "Situation-aware dynamic service coordination in an IoT environment," IEEE/ACM Transactions on Networking, vol. 25, no. 4, pp. 2082-2095, 2017.

[2] S. Wang, L. Tao, Q. Chen, J. Na, and X. Ren, "USDE-Based sliding mode control for servo mechanisms with unknown system dynamics," IEEE/ASME Transactions on Mechatronics, vol. 25, no. 2, pp. 1056-1066, 2020.

[3] Q. Chen, X. Yu, M. Sun, C. Wu, and Z. Fu, "Adaptive repetitive learning control of PMSM servo systems with bounded nonparametric uncertainties: theory and experiments," IEEE Transactions on Industrial Electronics, 2020.

[4] S. Wang, J. Na, X. Ren, H. Yu, and J. Yu, "Unknown input observer-based robust adaptive funnel motion control for nonlinear servomechanisms," International Journal of Robust and Nonlinear Control, vol. 28, no. 18, pp. 6163-6179, 2018.

[5] S. Wang, X. Ren, J. Na, and T. Zeng, "Extended-state-observer-based funnel control for nonlinear servomechanisms with prescribed tracking performance," IEEE Transactions on
Automation Science and Engineering, vol. 14, no. 1, pp. 98108, 2017.

[6] F. Qin, L. Wang, and S. Shao, "Emergy comparison of typical coastal urban ecosystems in china," Complexity, vol. 2020, Article ID 8071242, 7 pages, 2020.

[7] J. Zhang, X. Zhang, W. Wang et al., "Cache-enabled software defined heterogeneous networks for green and flexible $5 \mathrm{G}$ networks," IEEE Access, vol. 4, pp. 3591-3604, 2016.

[8] Q. Jian, "Overview of MAC protocols in wireless sensor networks," Journal of Software, vol. 19, no. 2, pp. 389-403, 2008.

[9] P. Joseph, J. Hill, and D. Culler, "Versatile low power media access for wireless sensor networks," in Proceedings of the 2nd International Conference on Embedded Networked Sensor Systems (SenSys'04), pp. 95-107, Baltimore, MD, USA, November 2004.

[10] M. Buettner, G. Yee, E. Anderson, and H. Richard, "X-MAC: a short preamble MAC protocol for duty-cycled wireless sensor networks," in Proceedings of the 4th International Conference on Embedded Networked Sensor Systems (SenSys'06), pp. 307-320, Colorado, USA, October 2006.

[11] Y. Sun, O. Gurewitz, and B. J. David, "RI-MAC: a receiver initiated asynchronous duty cycle MAC protocol for dynamic traffic loads in wireless sensor networks," in Proceedings of the 6th ACM Conference on Embedded Networked Sensor Systems (SenSys'08), pp. 1-14, Raleigh, NC, USA, November 2008.

[12] K. Chebrolu and A. Dhekne, "Esense: communication though energy sensing," in Proceedings of the 15th annual international conference on Mobile computing and networkingMobiCom '09, Beijing, China, September 2009.

[13] Y. Zhang and Q. Li, "HoWiES: a holistic approach to ZigBee assisted WiFi energy savings in mobile devices," in Proceedings of the IEEE INFOCOM'13, Turin, Italy, April 2013.

[14] X. Zhang and K. G. Shin, "Gap sense: lightweight coordination of heterogeneous wireless devices," in Proceedings of the IEEE INFOCOM'13, Turin, Italy, April 2013.

[15] S. M. Kim and T. He, "FreeBee: cross-technology communication via free side-channel," in Proceedings of the 21st Annual International Conference on Mobile Computing and Networking MobiCom '15, Paris, France, September 2015.

[16] Z. Yin, W. Jiang, S. M. Kim, and T. He, "C-morse: crosstechnology communication with transparent morse coding," in Proceedings of the IEEE Conference on Computer Communications, Atlanta, GA, USA, May 2017.

[17] Z. Chi, Z. Huang, Y. Yao, T. Xie, H. Sun, and T. Zhu, "EMF: Embedding multiple flows of information in existing traffic for concurrent communication among heterogeneous IoT devices," in Proceedings of the IEEE Conference on Computer Communications INFOCOM'17, Atlanta, GA, USA, October 2017.

[18] S. Yin, Q. Li, and O. Gnawali, "Interconnecting WiFi devices with IEEE 802.15.4 devices without using a gateway," in Proceedings of the 11th International Conference on Distributed Computing in Sensor Systems (DCOSS'15), pp. 127-136, Fortaleza, Brazil, July 2015.

[19] X. Guo, X. Zheng, and Y. He, "WiZig: cross-technology energy communication over a noisy channel," in Proceedings of the IEEE Conference on Computer Communications (INFOCOM'17), Atlanta, GA, USA, October 2017.

[20] Z. Chi, Y. Li, H. Sun, Y. Yao, Z. Lu, and T. Zhu, "B2W2: N-way concurrent communication for IoT devices," in Proceedings of the 14th ACM Conference on Embedded Network Sensor Systems, Stanford, CA, USA, September 2016. 
[21] Z. Li, Z. Yin, L. Liu, R. Liu, and He Tian, "WEBee: physicallayer cross-technology communication via emulation," in Proceedings of the 23rd Annual International Conference on Mobile Computing and Networking (Mobicom17), Snowbird, USA, October 2017.

[22] Z. Chi, Y. Li, Y. Yao, and T. Zhu, "PMC: parallel multiprotocol communication to heterogeneous IoT radios within a single WiFi channel," in Proceedings of the 25th International Conference on Network Protocols (ICNP'17), Toronto, ON, Canada, November 2017.

[23] S. Wang, N. Jing, and Y. Xing, "Adaptive optimal parameter estimation and control of servo mechanisms: theory and experiment," IEEE Transactions on Industrial Electronics, vol. 2020, Article ID 2141732, 45 pages, 2020.

[24] S. Wang and J. Na, "Parameter estimation and adaptive control for servo mechanisms with friction compensation," IEEE Transactions on Industrial Informatics, vol. 16, no. 11, pp. 6816-6825, 2020.

[25] F. Qin et al., "Kernel learning for blind image recovery from motion blur," Multimedia Tools and Applications, vol. 79, 2020.

[26] Q. Fu-Quang and Li F. Wang, "Effect of spatio-temporal change of land use on soil salinization in the Yellow River Delta," Desalination and Water Treatment, vol. 122, pp. 158-164, 2018.

[27] Qin, Q. Fu, and L. F. Wang, "Research on the estimation model of soil moisture content based on the characteristics of thermal infrared data," in Proceedings of the 12th International Conference on Complex, Intelligent, and Software Intensive Systems (CISIS), Matsue, Japan, June 2018.

[28] D. Yang, Y. Qiu, and S. Li, "RW-MAC: an asynchronous receiver-initiated ultra low power MAC protocol for wireless sensor networks," in Proceedings of the IET International Conference on Wireless Sensor Network, Beijing, China, November 2010.

[29] W. Sun, Z. Yang, X. Zhang, and Y. Liu, "Energy-efficient neighbor discovery in mobile ad hoc and wireless sensor networks: a surveyfficient neighbor discovery in mobile ad hoc and wireless sensor networks: a survey," IEEE Communications Surveys \& Tutorials, vol. 16, no. 3, pp. 1448-1459, 2014.

[30] H. Cai and T. Wolf, "On 2-way neighbor discovery in wireless networks with directional antennas," in Proceedings of the IEEE Conference on Computer Communications INFOCOM'15, pp. 1-9, Kowloon, Hong Kong, August 2015. 